\title{
Self-assembly of faceted Ni nanodots on $\mathrm{Si}(111)$
}

\author{
D. Aurongzeb, S. Patibandla, and M. Holtz ${ }^{\mathrm{a})}$ \\ Department of Physics, Texas Tech University, Lubbock, Texas 79409 \\ H. Temkin \\ Department of Electrical Engineering, Texas Tech University, Lubbock, Texas 79409
}

(Received 2 September 2004; accepted 18 January 2005; published online 2 March 2005)

\begin{abstract}
We report the formation of $\mathrm{Ni}$ nanodots on $\mathrm{Si}(111)$. Island density is varied by annealing temperature and time and is studied using atomic force microscopy (AFM) and magnetic force microscopy. Activation energies of $0.09 \pm 0.02$ and $0.31 \pm 0.05 \mathrm{eV}$ are observed for the formation of these islands. These are associated with Ni surface self-diffusion across the (111) and (110) Ni facets, respectively. For brief $500{ }^{\circ} \mathrm{C}$ anneals, regular nanodots are observed with self-limiting sizes of height $\sim 16 \mathrm{~nm}$ and area $180 \mathrm{~nm} \times 260 \mathrm{~nm}$, while density exhibits a power-law time dependence with exponent $1.13 \pm 0.12$. AFM analysis reveals a "truncated hut" shape consistent with (110) top and (111) sidewall surfaces. (C) 2005 American Institute of Physics. [DOI: 10.1063/1.1880452]
\end{abstract}

Nanometer-scale structures are of interest for applications in electronic, optoelectronic, and magnetic devices. Self-assembly has drawn considerable attention as an approach for producing nanometer-scale structures ${ }^{1,2}$ due to its relative simplicity when compared with conventional patterning techniques, such as focused ion beam, lithography, or template nanoimprinting. Formation of self-assembled structures generally depends on competition between the surface and interfacial free energies. Self-assembly of regular structures has been observed in numerous situations, particularly for semiconductor heteroepitaxy. ${ }^{3-5}$ Similar self-assembled growth has been recently reported for metal on metal ${ }^{6}$ and silicide on Si (Refs. 7 and 8) nanodot formation. Formation on self-assembled islands of $\mathrm{Ni}$, acting as a catalyst, is of interest in vapor-liquid-solid growth of semiconductor nanowires. ${ }^{9,10}$

In previous studies, ${ }^{4-8}$ films were deposited at a high substrate temperature to self assemble the nanodots. In this letter, we report a simple method for creating Ni nanodots of desired density. The approach relies on conventional postdeposition annealing. We find that $\mathrm{Ni}$ nanodots can be formed on $\mathrm{Si}(111)$ substrates provided a native oxide is present. The density can be varied according to anneal temperature and time, and for initial Ni thickness in a specific range. Annealing at $500{ }^{\circ} \mathrm{C}$ produces nanodots with regular size and shape. Interestingly, the nanodot formation is selflimited.

Nickel was deposited on $\mathrm{Si}(111)$ substrates using an electron-beam evaporator. The substrate cleaning included ultrasonically degreasing in acetone and methanol followed by a rinse with ultrapure water. This process preserves the native oxide. The $\mathrm{Ni}$ deposition was done at a rate of $\sim 1 \AA$ /s with a base pressure $<10^{-7}$ Torr with the substrate at room temperature. The deposition thickness ranges from 1 to $10 \mathrm{~nm}$, with the best nanodot formation occurring at $\mathrm{Ni}$ thickness of $3 \mathrm{~nm}$. After deposition, the samples were annealed at different temperatures and times inside a convection furnace. Annealing in $1 \mathrm{~atm} \mathrm{~N}_{2}$ or air played no observable role in the nanodot formation. The nanodot evolution was observed using atomic force and magnetic force mi-

\footnotetext{
${ }^{\text {a) }}$ Author to whom correspondence should be addressed; electronic mail: mark.holtz@ttu.edu
}

croscopies (AFM and MFM, respectively). Root-meansquare $(\mathrm{rms})$ roughness $\left(\sigma_{\mathrm{rms}}\right)$ was analyzed for the AFM measurements according to

$$
\begin{aligned}
\sigma_{\mathrm{rms}}^{2} & =\frac{1}{A} \iint_{A}(z(\mathbf{r})-\bar{z})^{2} d \mathbf{r} \\
& \rightarrow \frac{1}{(N-1)(M-1)} \sum_{i, j}^{N, M}\left(z_{i, j}-\bar{z}\right)^{2},
\end{aligned}
$$

where $z$ is the height at $\mathbf{r}=(x, y)$ coordinate, denoted $(i, j)$ in discretized form, and $\bar{z}$ is the mean height. For images analyzed here, $N=M=512$ in the images spanning area $A$ $=2 \mu \mathrm{m} \times 2 \mu \mathrm{m}$. Scanning electron microscopy was also used to examine the nanodots, although we do not focus on those images in this letter.

AFM measurements of as-deposited Ni films show very smooth surfaces, with $\sigma_{\text {rms }}<1 \mathrm{~nm}$. Figures $1(\mathrm{a})-1(\mathrm{~d})$ show AFM analyses of $\mathrm{Ni}$ nanodots formed following $500{ }^{\circ} \mathrm{C}$ annealing for several durations. The AFM images were obtained within $1 \mathrm{~h}$ of annealing. Studies showed the nanodots to relax from rectangular to approximately circular dome shapes after $\sim 1 \mathrm{~h}$ under ambient conditions. The $500{ }^{\circ} \mathrm{C}$
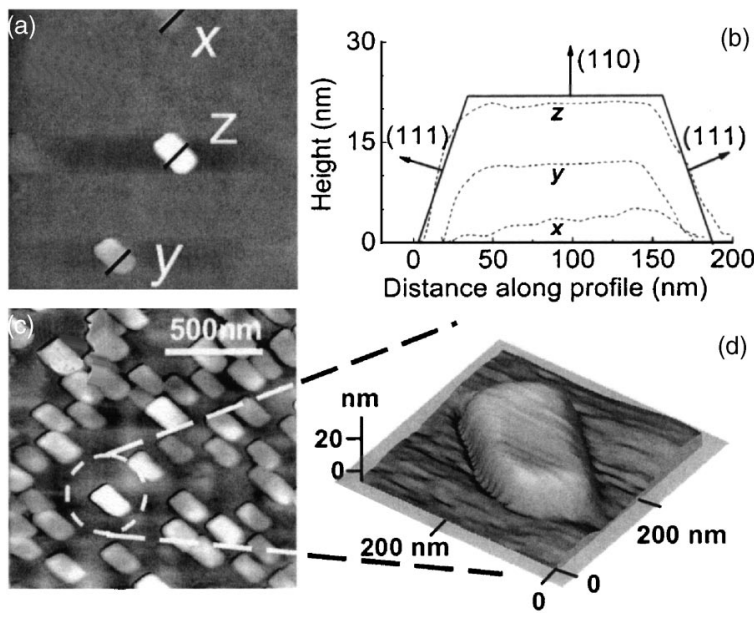

FIG. 1. Postanneal AFM image of nanostructures formed by $500{ }^{\circ} \mathrm{C}$ for (a) $35 \mathrm{~s}$, (b) AFM line images across nanodots captured at different stages of formation denoted $x, y$, and $z$ in (a), (c) $500{ }^{\circ} \mathrm{C}$ for $2 \mathrm{~min}$, and (d) expanded view of a well-formed nanodot in (c). Initial Ni thickness: $3 \mathrm{~nm}$. 


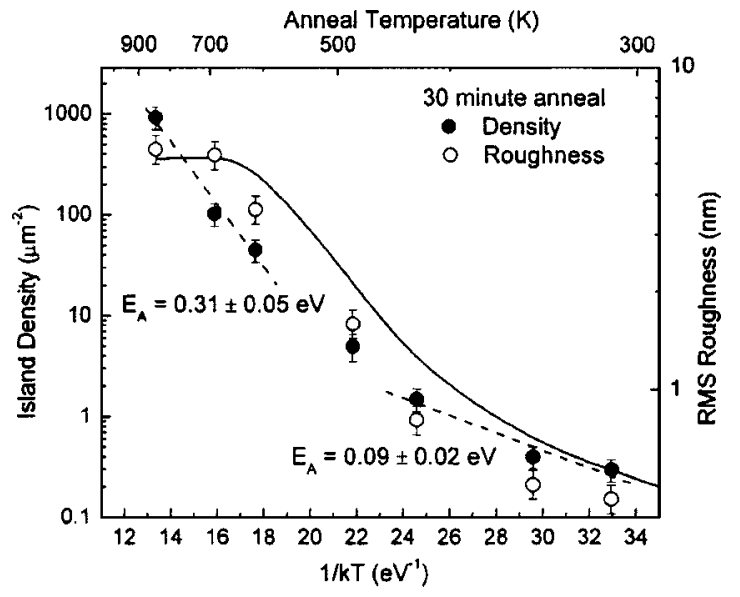

FIG. 2. Arrhenius plot of island density ( ). Activation energies are obtained from fits to the data in the low and high anneal temperature ranges (dashed lines). The rms roughness evolution is also shown $(\bigcirc)$. The solid curve is calculated rms roughness based on the density and uniform nanodot shapes.

anneals produced the truncated trapezoidal shapes shown in Fig. 1 with lateral dimensions $180 \pm 10 \mathrm{~nm}$ by $260 \pm 14 \mathrm{~nm}$ and thickness $16 \pm 5 \mathrm{~nm}$. Uncertainties are standard deviations. An enlarged view of a well-formed nanodot in Fig. 1(c) is shown in Fig. 1(d). The density is seen to increase with anneal time, but the sizes are found to be consistent, suggesting self-limited formation. Figure 1(b) shows line images across nanodots at different stages of formation in Fig. 1(a) $(x, y$, and $z)$. We discuss later the morphology of these structures.

Combined AFM and MFM images were obtained for island formation following $30 \mathrm{~min}$ anneals at several temperatures. Results are from images for fully relaxed nanodots, i.e., more than $1 \mathrm{~h}$ at room temperature following the annealing. AFM and MFM images showed the expected agreement. The island density $n$ is found to increase with anneal temperature, while nanodot sizes are approximately uniform up to the highest temperature studied, with lateral length scale $\sim 100 \mathrm{~nm}$ and height $h \sim 10 \mathrm{~nm}$. These images were collected after several hours and thus correspond to relaxed nanodots. Nanodots formed by annealing at $600{ }^{\circ} \mathrm{C}$ are substantially smaller, with lateral length scale $\sim 30 \mathrm{~nm}$. Figure 2 shows an Arrhenius plot of island density. The observed dependence is consistent with two activation energies. In the low anneal temperature range, we obtain $E_{A}$ $\sim 0.09 \pm 0.02 \mathrm{eV}$, while at higher temperatures the dependence yields $E_{A} \sim 0.31 \pm 0.05 \mathrm{eV}$. These activation energies are relatively low, suggesting surface diffusion as a likely mechanism for our Ni nanodot formation. Calculated values of Ni self-diffusion, across major crystal facets are $E_{A}(111)$ $=0.063 \mathrm{eV}, E_{A}(110)=0.39 \mathrm{eV}$, and $E_{A}(100)=0.68 \mathrm{eV} .{ }^{11}$ Our measured $E_{A}$ results are in reasonable agreement with the calculated values for activated surface diffusion of $\mathrm{Ni}$ on (111) and (110) surfaces. The formation kinetics are thus consistent with diffusion-driven growth primarily across these two crystal surfaces.

Returning to the anneal time dependence at $500{ }^{\circ} \mathrm{C}$, two aspects are interesting. First, the island density increases with anneal time. Second, the size and shape of the structures does not appear to depend strongly on anneal time, with slightly taller nanodots formed by the longest anneal duration. Figure 3 shows the island density versus anneal time on Downloaded 03 Mar 2005 to 129.118.86.82. Redistribution subject

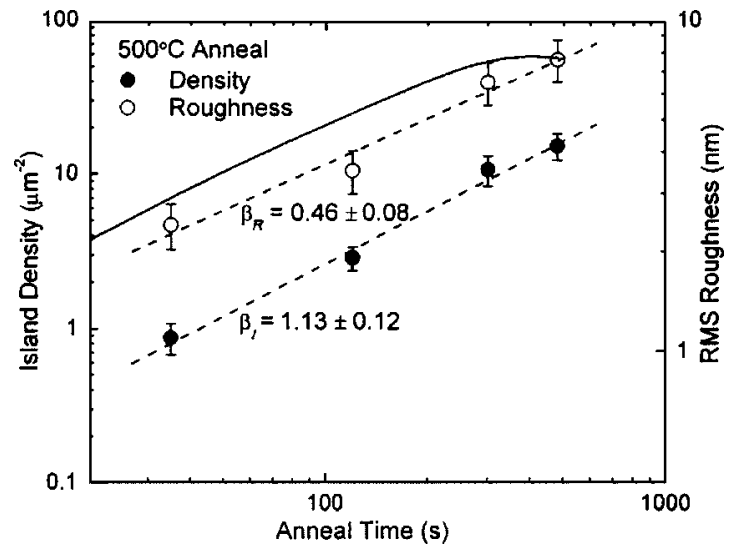

FIG. 3. Time dependence of the island density $(\bullet)$ and rms roughness $(\bigcirc)$. The dashed lines illustrate the power law behavior with exponents shown. The solid curve is calculated rms roughness based on the density and uniform nanodot shapes. Initial Ni thickness: $3 \mathrm{~nm}$.

a $\log$-log scale. We observe a linear dependence such that $n \propto t^{\beta}$, where $t$ is the anneal time and $\beta$ is the growth exponent. The observed growth is consistent with a power-law behavior with island growth exponent $\beta_{I}=1.13 \pm 0.12$. This value is consistent with $1.29 \pm 0.07$ reported for $\mathrm{ErSi}_{2}$ nanodot ripening on silicon, ${ }^{8}$ which was interpreted as illustrating a direct dependence with time. The growth of our $\mathrm{Ni}$ nanodots is likewise interpreted as being in proportion to anneal time, although the formation is not a ripening process. We do not observe the island density saturation expected $\sim 200 \mathrm{~s}$. It is possible that the additional volume apparent in the nanodots arises from $\mathrm{Si}$ diffusion into the region. An elevated AFM tip radius could also contribute to the apparent volume, the tips used here have a nominal tip radius of $10 \mathrm{~nm}$. We will return to the nanodots volume when we discuss nucleation of the nanodots.

Figure 2 includes rms roughness versus $1 / k T$ and Fig. 3 graphs $\sigma_{\text {rms }}$ versus anneal time at $500{ }^{\circ} \mathrm{C}$. rms roughness values were determined from the AFM images. In each case, $\sigma_{\text {rms }}$ increases with island density. A direct connection between the island area density and $\sigma_{\text {rms }}$ is found as follows. We assume isolated structures having uniform shape with area $A_{0}$ and height $h$. The dimensionless area fill factor is defined as $f_{A}=n A_{0}$. Using the definition of rms roughness (integral form), we obtain by straightforward integration

$$
\sigma_{\text {rms }}=\sqrt{n A_{0}\left(1-n A_{0}\right)} h .
$$

Using the island density dependence in the respective graphs and $h$ from the AFM images, we arrive at the solid curves shown in Figs. 2 and 3. The good agreement between the measured $\sigma_{\text {rms }}$ and this straightforward analysis supports, on statistical grounds, the uniformity in our nanodot formation. In Fig. 3, the roughness is also well fit by a linear dependence such that $\sigma_{\text {rms }} \propto t^{\beta}$. The exponent obtained from linear fit is $\beta_{R}=0.46 \pm 0.08$. This value is consistent with what is expected from Eq. (2), in the low $f_{A}$ limit, when $n \propto t$ and $A_{0}$ is constant. It is also consistent with what is expected for interface evolution in the presence of random pinning forces. ${ }^{12}$ In the current context, the randomness is related to the nucleation sites where the nanodots form, while the pinning is attributed to $\mathrm{Ni}$ atoms being trapped by nanodots once they arrive by surface diffusion. This interpretation is consistent with the fact that we do not observe ripening. to AIP license or copyright, see http://apl.aip.org/apl/copyright.jsp 
We now discuss the self-limiting behavior. Figure 1(a) shows an AFM image following a very brief anneal at $500{ }^{\circ} \mathrm{C}$. Some islands are already well-formed, resembling the islands formed after longer anneals [Fig. 1(c)]. Additionally, we capture several islands in the early stages of formation [denoted by scan lines $x, y$, and $z$ in Fig. 1(a)]. In Fig. 1(b), we show representative AFM line profiles across islands at different levels of development. Each island exhibits a similar lateral extent. For fully formed structures, the top surfaces are flat, and the sidewalls make an angle of $\sim 37^{\circ}$ with the base. This shape is highly repeatable. In the spirit of Ref. 6, we also include in Fig. 1(b) a sketch of the "truncated hut" shape we propose based on the AFM images. The shape of our fully formed structures is consistent with a top surface with $\mathrm{Ni}$ (110) facet and (111) sidewalls. This assignment is also in accord with the two surface diffusion activation energies obtained from our kinetics studies, which were consistent with $E_{A}(111)$ and $E_{A}(110)$. This agreement suggests that the formation process involves diffusion across these surfaces. Because the (111) surface has lower surface energy ${ }^{13}$ and lower surface diffusion activation energy, ${ }^{11}$ it forms more rapidly than the (110) surfaces, which only fully form in the final stages.

The presence of the native oxide plays a key role in the nanodot formation. We have attempted to form Ni nanodots using the same procedures but on $\mathrm{Si}(111)$, which has been stripped of oxide, and on $\operatorname{Si}(111)$ with a thermal oxide $(100 \mathrm{~nm})$. In both cases, uniform properties are obtained and Ni nanodots are not observed. Native oxides are known to be nonuniform in coverage. Since Ni can diffuse through voids in $\mathrm{SiO}_{2},{ }^{14,15}$ a patchy native oxide layer will permit this diffusion, and subsequent $\mathrm{NiSi}_{2}$ growth. ${ }^{16}$ We suggest here that formation occurs where the native oxide is thin. In the $500{ }^{\circ} \mathrm{C}$ temperature range, $\mathrm{Ni}$ diffusion through the oxide layer and agglomeration on the surface compete. ${ }^{15}$ As established here, surface diffusion of $\mathrm{Ni}$ on $\mathrm{Ni}$ is the primary formation mechanism. Once the $\mathrm{NiSi}_{2}$ nucleation sites form, $\mathrm{Ni}$ atoms diffusing across the surface will tend to agglomerate at these places contributing to the nanodot formation. The MFM measurements confirm that the surfaces of our nanodots are magnetic. It is possible that $\mathrm{Si}$ will diffuse from the bulk into the regions where $\mathrm{NiSi}_{2}$ has formed, since extended defects occur in the nearby silicon. These extended defects act as fast diffusion paths for the silicon. ${ }^{17}$ Thus, it is possible that some of the elevation seen in our nanodots stems from this local enhancement of silicon and the volume expansion inherent in silicide formation.

We now discuss factors relevant to the self-limited growth. Initially, formation occurs with predominantly (111) and (110) $\mathrm{Ni}$ surfaces, due to their lower formation energies. ${ }^{13}$ The former grow, but diffusion tends to enhance the (110) formation. This is because $\mathrm{Ni}$ atoms readily traverse the (111) surfaces, and are slowed when they arrive at a (110) facet. The (110) and (111) surfaces are prevalent in the equilibrium structures, Fig. 1. As growth proceeds, nearby reservoirs of $\mathrm{Ni}$ atoms are depleted, contributing to the self-limiting behavior. The highly regular shapes observed in Fig. 1 suggest that stress may play a role in the nanodot formation. ${ }^{78}$ In our current picture, the substratenanodot stress would primarily originate at the $\mathrm{Ni} / \mathrm{NiSi}_{2}$ interface. Lattice constant mismatches between these materials are enormous, $\sim 35 \%,{ }^{6}$ so that epitaxy is impossible. Furthermore, we do not know how the lateral extent of the $\mathrm{NiSi}_{2}$ compares with the observed nanodots. Application of the Tersoff and Tromp model, ${ }^{3}$ relating surface and interface energies to optimal conformation, suggests $\sim 1 \%$ mismatch strain between the $\mathrm{Ni}$ and the underlying material. The observation that relaxation into dome shapes occurs after several hours indicates that the surface and interface energies are comparable. That is, the Ni nanodots formed at $500{ }^{\circ} \mathrm{C}$, shown in Fig. 1, are close to the maximum size achievable with a rectangular shape. For comparison, the stable Ni nanodots formed on TiN are $\sim 25 \mathrm{~nm}$ across, ${ }^{6}$ considerably smaller than what we report here. In contrast, the $\mathrm{ErSi}_{2}$ (Ref. 7) and $\mathrm{TiSi}_{2}$ (Ref. 8) nanodots on $\mathrm{Si}(111)$ substrates are elongated. It was concluded that the mismatch strains, in these two cases, were smaller than expected due to the formation of extended defects. Given the anistropic nature of the surface used in preparing our nanodots, it is possible that extended defects are important here as well in mitigating the mismatch strain between $\mathrm{Ni}$ and $\mathrm{NiSi}_{2}$.

In summary, we have prepared magnetic $\mathrm{Ni}$ nanodots using a straightforward, postdeposition annealing approach. Activation energies are determined and related to surface diffusion of Ni across (111) and (110) Ni facets. Detailed studies in the $500{ }^{\circ} \mathrm{C}$ anneal temperature range reveal regular shapes with self-limited sizes. The structures are found to have regular truncated hut shapes consistent with (110) top and (111) sidewall surfaces. These structures are observed to relax into stable dome shapes after $\sim 1 \mathrm{~h}$ in ambient conditions, suggesting that stress plays a role in the shape along with the formation diffusion kinetics and Ni reservoir depletion. The nanodots do not coalesce. This can be attributed to the Ni depletion, and also, in the regular structures, the presence of strain-field repulsion ${ }^{18}$ which will be greater near their bases. ${ }^{19}$

The authors acknowledge support of this research by the National Science Foundation (CTS-0210141 and ECS0304224).

${ }^{1}$ H. Omi and T. Ogino, Appl. Phys. Lett. 71, 2163 (1997).

${ }^{2}$ J. Nogami, B. Z. Liu, M. V. Katkov, C. Ohbuchi, and N. O. Birge, Phys. Rev. B 63, 233305 (2001).

${ }^{3}$ F. M. Ross, J. Tersoff, and R. M. Tromp, Phys. Rev. Lett. 80, 984 (1998).

${ }^{4}$ G. Medeiros-Ribeiro, T. I. Kamins, D. A. A. Ohlberg, and S. R. Williams, Phys. Rev. B 58, 3533 (1998).

${ }^{5}$ A. Rastelli, H. von Kanel, B. J. Spencer, and J. Tersoff, Phys. Rev. B 68, 115301 (2003)

${ }^{6}$ H. Zhou, D. Kumar, A. Kvit, A. Tiwari, and J. Narayan, J. Appl. Phys. 94, 4841 (2003).

${ }^{7}$ L. Fitting, M. C. Zeman, W.-C. Yang, and R. J. Nemanich, J. Appl. Phys. 93, 4180 (2003)

${ }^{8}$ W.-C. Yang, H. Ade, and R. J. Nemanich, J. Appl. Phys. 95, 1572 (2004).

${ }^{9}$ J. Zhang and L. Zhang, J. Vac. Sci. Technol. B 21, 2415 (2003).

${ }^{10}$ G. Kipshidze, B. Yavich, A. Chandolu, J. Yun, V. Kuryatkov, D. Aurongzeb, I. Ahmad, M. Holtz, and H. Temkin, Appl. Phys. Lett. 86, 033104 (2005).

${ }^{11}$ U. Kürpick, Phys. Rev. B 64, 075418 (2001).

${ }^{12}$ H. Leschhorn, Physica A 195, 324 (1993).

${ }^{13}$ L. Vitos, A. V. Ruban, H. L. Skriver, and J. Kollár, Surf. Sci. 411, 186 (1998).

${ }^{14}$ H. Dallaporta, M. Liehr, and J. E. Lewis, Phys. Rev. B 41, 5075 (1990).

${ }^{15}$ J. O. Olowolafe, M.-A. Nicolet, and J. W. Mayer, Thin Solid Films 38, 143 (1976).

${ }^{16}$ R. T. Tung, J. M. Gibson, and J. M. Poate, Phys. Rev. Lett. 50, 429 (1983).

${ }^{17}$ D. Jones and V. Palermo, Appl. Phys. Lett. 80, 673 (2002).

${ }^{18}$ A. Ponchet, D. Lacombe, L. Durand, D. Alquier, and J.-M. Cardonna, Appl. Phys. Lett. 72, 2984 (1998).

${ }^{19}$ D. E. Jesson, T. P. Munt, V. A. Shchukin, and D. Bimberg, Phys. Rev. Lett. 92, 115503 (2004). 